\title{
Targeting heme oxygenase- 1 in early diabetic nephropathy in streptozotocin-induced diabetic rats
}

\author{
R Abo El Gheit, MN Emam \\ Physiology Department, Faculty of Medicine, Tanta University, Tanta, Egypt
}

Received: August 29, 2015

Accepted: May 11, 2016

\begin{abstract}
Diabetic nephropathy (DN) is one of the most common microvascular diabetic complications. This study was designed to evaluate the possible protective effect and underlying mechanisms of HO-1 induction in streptozotocin (STZ)-induced early DN in rats. The diabetic rats were divided into three groups: STZ-diabetic, cobalt protoporphyrin (CoPP)-treated diabetic, and zinc protoporphyrin IX (ZnPP)-treated diabetic groups. Compared to the STZdiabetic group, CoPP-induced HO-1 upregulation improved the diabetic state and renal functional parameters, suppressed the renal proinflammatory marker, NF- $\kappa \mathrm{B}$, abrogated the elevated renal hydroxyprolin, and decreased the enhanced renal nicotinamide adenine dinucleotide phosphate oxidase activity with parallel reduction of urinary oxidative stress markers. On the contrary, treatment with $\mathrm{ZnPP}$ abrogated HO-1 levels, aggravated the diabetic condition with further increases in renal oxidative stress, fibrotic and inflammatory markers, and exacerbated renal dysfunction in diabetic animals. These findings suggest that the reduced diabetic renal injury upon HO-1 induction implicates the role of HO-1 induction as a potential treatment for DN.
\end{abstract}

Keywords: diabetic nephropathy, heme oxygenase, cobalt protoporphyrin, zinc protoporphyrin IX, renal hydroxyprolin

\section{Introduction}

Diabetes mellitus is a multisystem disorder that affects various organs. Almost $30 \%$ of diabetic patients develop diabetic nephropathy (DN) despite control of blood glucose and/or blood pressure (47). The underlying molecular mechanisms of DN are not fully established. DN has been traditionally considered to be caused by metabolic and hemodynamic alterations but recent studies suggest that inflammatory processes with chronic low grade inflammation and aberrant immune responses are also involved in the development and progression of the disease (18).

Increased levels of some proinflammatory factors, such as the intercellular adhesion molecule (ICAM-1) and monocyte chemoattractant protein (MCP-1) have been found in diabetic patients with nephropathy. MCP-1 and ICAM-1 have been identified as key players in monocyte/macrophage infiltrations and leukocyte adhesion in diabetic animal models (52).

A common denominator of diabetes is the enhanced level of tumor necrosis factor- $\alpha$ (TNF- $\alpha$ ), which has been largely implicated in the inflammatory cascade, which in turn activates the nuclear factor kappa-B (NF- $\mathrm{B}$ ) pathway, creating a vicious cycle that exacerbates diabetes and its renal complications (63).

Corresponding author: Rehab Abo El Gheit

Physiology Department, Faculty of Medicine, Tanta University

Mohamed Adel Salam Awara Street, Tanta 31511, Egypt

Phone: +20 127 6640441; Fax: +20115 3203200; E-mail: extrasystole.2003@gmail.com 
Long-standing hyperglycemia, a common cardinal feature in diabetes, is known to stimulate renal cells to produce several humoral mediators and growth factors (54). These factors form complex cross-links over time and induce several structural alterations such as interstitial extracellular matrix expansion that is initially triggered by increased cellular components, followed by an increase in interstitial fibrillar collagen (55) that appears to be critical for final progression of DN (21).

Increased oxidative stress has been implicated in the pathogenesis of diabetes. Nicotinamide adenine dinucleotide phosphate oxidase (NADPH), the major source of superoxide production, has been shown to be activated in the kidney of diabetic animal models with enhanced expression in the glomerulus and distal tubules, constituting a fundamental link between hyperglycemia and oxidative stress that ultimately leads to deterioration of renal function (17).

Heme oxygenase (HO) is a ubiquitous microsomal rate-limiting enzyme involved in the oxidative degradation of heme to biliverdin (BV), which is rapidly converted into bilirubin (BR) by BV reductase. During this step, iron is released from the heme ring and carbon monoxide (CO) is generated (2). To date, three distinct isoforms of $\mathrm{HO}$ have been identified: HO-1, an inducible form; HO-2, a constitutive form; and HO-3, probably a pseudo gene (2).

Among these isoforms, HO-1 has been the most extensively studied HO isoenzyme. Increased HO-1 expression seems to provide cytoprotection and HO-1 is known to be upregulated in the kidney under various physical, chemical, and pathophysiological stimuli including oxidative and inflammatory insults, as well as metabolic and hemodynamic factors such as high glucose, elevated blood pressure, and lipids (27).

$\mathrm{HO}-1$ induction is considered to be an adaptive cellular response to stress. Therefore, HO-1 may be considered a sensitive index that is triggered with the onset of pathophysiological changes. The pathophysiological activation of HO-1 may fall below the threshold necessary to activate important components through which the HO system can restore tissue homeostasis. Stronger effects can be achieved by some pharmacological agents capable of inducing some HO, like metalloprotoporphyrins such as cobalt protoporphyrin (CoPP) (39).

The importance of HO-1 in altering the outcome of many diseases is established by observations that pharmacological induction or overexpression of HO-1, as well as administration of the different end-products of heme catabolism all have significant beneficial or therapeutic effects in a large number of pathologic conditions (27).

Although many therapeutic interventions have been shown to delay the development or retard the progression of $\mathrm{DN}$, currently no intervention has been able to halt or reverse its progression (29). Therefore, better therapeutic modalities are urgently needed. The HO may constitute a novel system that could be explored against diabetes and its related renal and metabolic complications. This study highlights the potential therapeutic benefit of HO-1 induction by CoPP to protect the kidney from diabetic renal injury.

\section{Materials and Methods}

\section{Animals}

Forty male albino rats weighing 150-180 g were used after 1 week of proper acclimatization to the animal house conditions ( $12 \mathrm{~h}$ lighting cycle and $25 \pm 2{ }^{\circ} \mathrm{C}$ temperature). The rats were housed four per cage, had free access to standard rodent chow and water. Procedures involving animals and their care were conducted in conformity with the protocols of the Research Advisory Ethical Committee of Faculty of Medicine, Tanta University, Egypt. 


\section{Experimental induction of diabetes}

Diabetes was induced in overnight fasted rats by a single i.p. injection of freshly prepared streptozotocin (STZ) $(60 \mathrm{mg} / \mathrm{kg}$, dissolved in $0.1 \mathrm{M}$ cold citrate buffer, $\mathrm{pH} 4.5)$ (10). STZ was purchased from Sigma-Aldrich (St. Louis, MO, USA). The STZ-treated animals were allowed to drink 5\% glucose solution instead of drinking water for the first $24 \mathrm{~h}$ after STZ challenge to overcome initial drug-induced hypoglycemic mortality. Three days after STZ injection, blood samples were collected and blood glucose levels were measured using a glucometer (OneTouch Horizon, LifeScan, Johnson \& Johnson, CA, USA). Animals with blood glucose level above $250 \mathrm{mg} / \mathrm{dl}$ were used. After the maintenance for 4 weeks, the blood and urine samples from these rats were again tested for hyperglycemia and proteinuria. The urine protein was estimated by Biuret's method using a commercial kit (Diamond Diagnostic, Egypt). Rats with hyperglycemia ( $\geq 250 \mathrm{mg} / \mathrm{dl}$ ) and proteinuria $(\geq 8.0 \mathrm{mg} / \mathrm{dl})$ at the end of 4 weeks post STZ injection were selected for further study.

\section{Experimental procedures}

The diabetic rats were left untreated, for 4 weeks after induction of diabetes, to induce early DN $(48,50)$ and were assigned to receive treatments with either CoPP or zinc protoporphyrin IX (ZnPP). The animals were divided into four groups of 10 rats each. The control normal group received i.p. injections of $0.1 \mathrm{~mol} / 1$ citrate buffer, $\mathrm{pH} 4.5$ (vehicle for STZ); the diabetic group was STZ-injected and vehicle-treated, the CoPP-treated diabetic group received CoPP at $15 \mathrm{mg} / \mathrm{kg}$ i.p. (Sigma-Aldrich, UT, USA), and the ZnPP-treated diabetic group was injected with ZnPP at $4 \mu \mathrm{mol} / \mathrm{kg}$ i.p. (Sigma-Aldrich, UT, USA). Both CoPP and ZnPP were administered twice weekly for 4 weeks.

At the end of 8 weeks after STZ injection, the rats were placed in metabolic cages (Nalgene Corp., Rochester, NY, USA) for 24-h urine collection. Blood samples were collected after overnight fasting and centrifuged at 3,000g for $10 \mathrm{~min}$ to obtain clear sera. Then the kidneys of all rats were immediately excised, decapsulated, and divided longitudinally into two equivalent sections. One section was retained with dissection of the renal cortices that were snap frozen in liquid nitrogen, stored at $-80{ }^{\circ} \mathrm{C}$, and subsequently homogenized in cold potassium phosphate buffer $(0.05 \mathrm{M}, \mathrm{pH} 7.4)$ for various biochemical analyses. The protein content of the renal samples was measured by the method of Bradford (3), using crystalline bovine serum albumin as standard.

Assessment of STZ-induced diabetic state

Using commercially available colorimetric kits, serum glucose (Diamond Biodiagnostic Comp., Egypt) was estimated. Serum insulin was estimated using a rat insulin ELISA kit (American Diagnostica Inc., San Francisco, CA, USA).

\section{Assessment of STZ-induced DN}

Serum and urinary creatinine levels were estimated by standard alkaline picrate method using commercially available creatinine estimation kit (Cayman Chemical, Ann Arbor, MI, USA) $(57,58)$. Urinary albumin was measured by ELISA using microalbumin estimation kit (BioSystems, Spain), and collagen (Exocell, Philadelphia, PA, USA) excretion levels were determined as indices of renal injury.

Urinary 8-isoprostane and 8-hydroxydeoxyguanosine (8-OHdG) assay

Urinary 8-isoprostane, as a marker of lipid peroxidation, was estimated by ELISA as described by Milne et al. (31), using kit purchased from Cayman Chemical (Ann Arbor, MI, USA). 
Urinary 8-OHdG, as a marker of oxidative DNA damage, was estimated as described by Kim et al. (23). The concentration of urinary 8-OHdG was calculated from the standard curve and divided by the urinary creatinine.

\section{Renal nuclear protein extraction and $N F-\kappa B$ p65 subunit assay}

Nuclear protein was isolated from the kidneys as described by Lee et al. (25) and Zhou et al. (66). The nuclear level of $\mathrm{p} 65$ correlates positively with the activation of NF-kB pathway (64). The NF-кB/p65 ActivELISA (Imgenex, San Diego, CA, USA) kit was used to quantify $\mathrm{NF}-\kappa \mathrm{B}$ free $\mathrm{p} 65$ in the nuclear fraction of kidney tissue homogenate. The analysis was done according to the manufacturer's instructions.

\section{Renal NADPH oxidase activity and TNF- $\alpha$ level}

NADPH activity was measured in renal cortical samples as previously described (35). Average sample counts per minute (cpm) were normalized to $\mu \mathrm{g}$ protein (cpm/ $\mu \mathrm{g}$ protein). Renal TNF- $\alpha$ assay was performed with rat TNF- $\alpha$ ELISA kit (RayBiotech, Inc., GA, USA) according to the supplier's instructions.

Renal HO-1 activity and hydroxyproline level assay

HO-1 activity was measured in renal cortical samples using a commercially available ELISA according to the manufacturer's instructions (Enzo Life Sciences Inc., Farmingdale, NY, USA). Hydroxyproline level, as an index of basement membrane collagen, was measured according to the method described by Woessner (62).

\section{Renal cortical MCP-1 and soluble ICAM-1 assays}

Renal cortical MCP-1 levels were assessed using a commercially available ELISA according to the manufacturer's instructions (BD Biosciences, Bedford, MA, USA) (10). Renal soluble ICAM-1 levels were also determined using a commercially available ELISA according to manufacturer's instructions (R\&D Systems, Minneapolis, MN, USA) (45).

\section{Statistical analysis}

The data are expressed as mean \pm standard deviation (SD). Statistical analysis was performed by one-way analysis of variance to test any differences among the mean values of all groups. If differences were established, the values of two groups were compared with by multiple $t$ tests followed by Bonferroni correction. Group differences at the level of $P<0.05$ were considered statistically significant. Analyses were performed using GraphPad Prism version 4.0 software (GraphPad Software, San Diego, CA, USA).

\section{Results}

Effect of CoPP on serum insulin and glucose levels in STZ-diabetic rats Induction of diabetes with STZ resulted in marked hyperglycemia associated with significant reduction in insulin level (Fig. 1). CoPP induced HO upregulation in STZ-diabetic rats resulted in significant lowering in fasting glucose level that coincided with increased levels of plasma insulin, while ZnPP therapy exacerbated hyperglycemia, and reduced insulin levels compared to STZ-diabetic rats. 

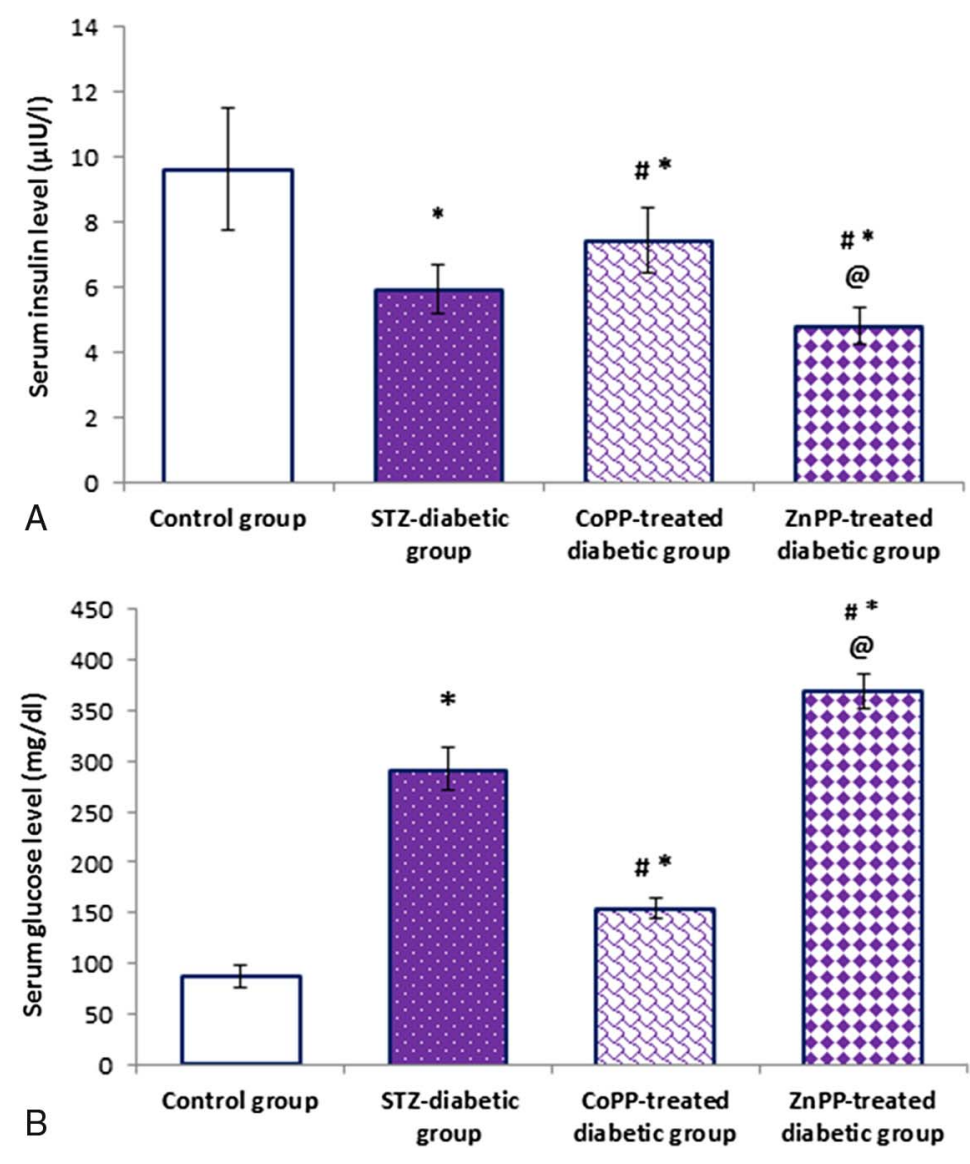

Fig. 1. Effects of the $\mathrm{HO}$ inducer, CoPP and $\mathrm{HO}$ inhibitor, $\mathrm{ZnPP}$ on serum insulin (A) and fasting (overnight fasting) glucose (B) levels in STZinduced early DN in rats. Data are mean \pm SD of 10 rats.

$* P<0.05$ vs. the control group;

${ }^{\#} P<0.05$ vs. the STZdiabetic group; ${ }^{\circledR} P<0.05$ vs. the CoPP-treated diabetic group

Effect of CoPP on renal functions in STZ-diabetic rats

Eight weeks after induction of diabetes with STZ, the diabetic rats exhibited marked deterioration in renal functions (Fig. 2), as indicated by a significant elevation in serum creatinine level $(1.648 \pm 0.262 \mathrm{vs} .0 .462 \pm 0.097 \mathrm{mg} / \mathrm{dl})$ associated with increased creatinine excretion ( $32.889 \pm 4.149$ vs. $8.433 \pm 1.123 \mathrm{mg}$ /day). Marked albuminuria $(5.304 \pm 0.578$ vs. $0.402 \pm 0.122 \mathrm{mg} /$ day) and marked increase in collagen excretion (46.276 \pm 4.642 vs. $14.939 \pm 0.977 \mu \mathrm{g} /$ day) was observed in diabetic rats compared to the control group (Fig. 2). Treatment with the HO inducer, CoPP resulted in marked improvement in renal parameters as indicated by significant reduction in serum creatinine $(0.826 \pm 0.174 \mathrm{mg} / \mathrm{dl})$ and urinary creatinine $(18.919 \pm 0.978 \mathrm{mg} /$ day $)$, albumin $(2.202 \pm 0.635 \mathrm{mg} /$ day $)$, and collagen excretion $(21.467 \pm 1.898 \mu \mathrm{g} /$ day $)$ compared to STZ-diabetic animals, while HO inhibitor, ZnPP further deteriorated the renal parameters.

Effect of CoPP on renal MCP-1, ICAM-1, TNF- $\alpha$, and NF-кB in STZ-diabetic rats

The STZ-diabetic rats exhibited significant increase in the renal levels of ICAM-1, MCP-1, and TNF- $\alpha$ (Fig. 3). Interestingly, the administration of CoPP therapy to STZ-diabetic rats significantly abated the STZ-induced elevated renal ICAM-1, MCP-1, and TNF- $\alpha$ expression, whereas administration of $\mathrm{ZnPP}$ to diabetic rats further aggravated these 

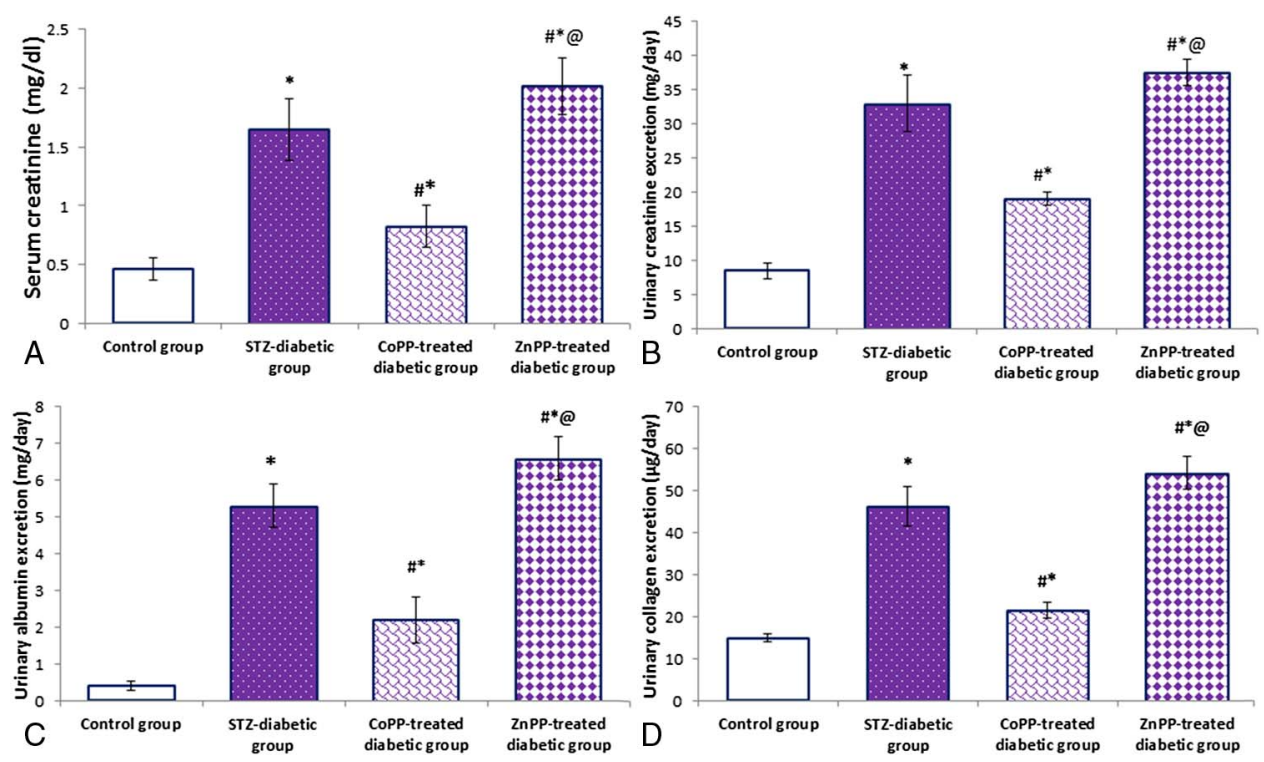

Fig. 2. Effects of the $\mathrm{HO}$ inducer, $\mathrm{CoPP}$ and $\mathrm{HO}$ inhibitor, $\mathrm{ZnPP}$ on serum creatinine (A), urinary creatinine (B), albuminuria (C), and collagen excretion (D) in STZ-induced early DN in rats. Data are mean \pm SD of 10 rats. ${ }^{*} P<0.05$ vs. the control group; ${ }^{\#} P<0.05$ vs. the STZ-diabetic group; ${ }^{@} P<0.05$ vs. the CoPP-treated diabetic group
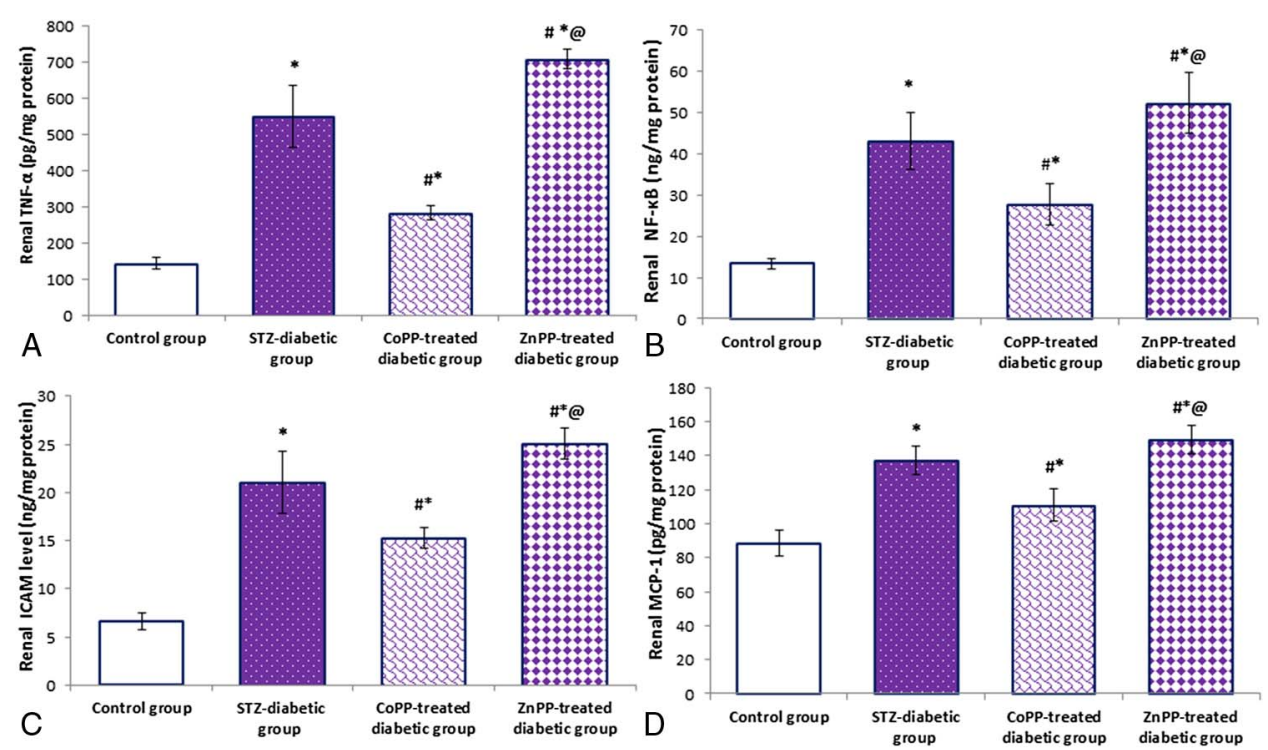

Fig. 3. Effects of the HO inducer, CoPP and HO inhibitor, ZnPP on renal proinflammatory makers, renal TNF- $\alpha$ (A), NF- $\mathrm{BB}(\mathrm{B})$, ICAM-1 (C), and MCP-1 (D) in STZ-induced early DN in rats. Data are mean \pm SD of 10 rats. ${ }^{*} P<0.05$ vs. the control group; ${ }^{\#} P<0.05$ vs. the STZ-diabetic group; ${ }^{\circledR} P<0.05$ vs. the CoPP-treated diabetic group. ICAM-1 = intercellular adhesion molecule; MCP-1 = monocyte chemoattractant protein; TNF- $\alpha=$ tumor necrosis factor- $\alpha$; NF- $\kappa \mathrm{B}=$ nuclear factor kappa-light-chain enhancer of activated $\mathrm{B}$ 
proinflammatory markers. The STZ-induced diabetes was associated with marked increase in renal NF- B compared to normal control group. CoPP therapy markedly attenuated the elevated NF- $\mathrm{KB}$ in diabetic rats $(27.79 \pm 5.067$ vs. $43.17 \pm 6.886 \mathrm{ng} / \mathrm{mg}$ protein $)$. On the other hand, ZnPP administration to diabetic rats exacerbated the elevated NF- $\mathrm{kB}$.

\section{Effect of CoPP on renal NADPH oxidase and oxidative stress biomarkers}

Renal cortical NADPH oxidase activity was significantly elevated in STZ-diabetic rats compared to normal control group (Fig. 4). The increased NADPH oxidase activity was also associated with elevated urinary oxidative stress markers, 8-isoprostane, and 8-OHdG excretion levels in STZ-diabetic vs. the control group. Induction of HO-1 with CoPP significantly inhibited NADPH oxidase activity and reduced urinary excretion levels of 8 -isoprostane and 8-OHdG in diabetic rats by 1.4-, 1.5-, and 1.7-fold, respectively, whereas $\mathrm{ZnPP}$ application resulted in further accentuation in renal NADPH oxidase, urinary 8-isoprostane, and 8-OHdG by 1.2-, 1.1-, and 1.2-fold compared to STZ-diabetic animals, suggesting that induction of HO-1 displayed antioxidant capacity in diabetic kidney.

\section{Effect of CoPP on renal HO-1 activity in STZ-induced diabetic rats}

Our results demonstrated that STZ 1.2-fold upregulated HO-1 activity in diabetic kidneys compared to control normal kidneys. CoPP therapy induced further upregulation of HO-1 activity by 1.6 -fold, while $\mathrm{ZnPP} 1.7$-fold reduced HO-1 activity compared to the diabetic group (Fig. 5).

\section{Effect of CoPP on renal hydroxyproline level in STZ-induced diabetic rats}

The renal hydroxyproline level was significantly higher in STZ-induced diabetic rats compared to the control group (Fig. 6). Induction of HO-1 with CoPP markedly reduced the elevated renal hydroxyproline content by about 1.6-fold, an effect which was reversed by $\mathrm{ZnPP}$ treatment. These results suggest that HO-1 upregulation could efficiently prevent renal fibrosis in diabetic rats.

\section{Discussion}

HO-1 may be protective against stress-associated physiological disorders on the basis of its rapid upregulation under various stress conditions and potent physiological regulating properties (27).

In the present study, we validated the efficacy of HO-1 induction in the treatment of DN and the blockade of its associated signaling pathways underlying the pathophysiology of DN.

Many studies have underscored the cytoprotective effects of the HO system (7, 9, 10, 27). The mechanisms underlying the renoprotective effect of CoPP in diabetic rats are complex, challenging, and not fully understood. Substantial evidence indicates that HO-1 provides the provenance of pathways that can interrupt virtually all major mechanisms of diabetic kidney injury, including reduction of the high blood glucose that represents the central core of DN $(32,36,38)$.

The results obtained in our study strengthened the antidiabetic role of HO-1 induction in STZ-induced diabetes (Fig. 1), as evidenced by a significant insulinotropic effect that was associated with lowering of glucose level. Our data are consistent with those previously published in different diabetic models, including the nonobese Goto-Kakizaki (GK) rats (38), 

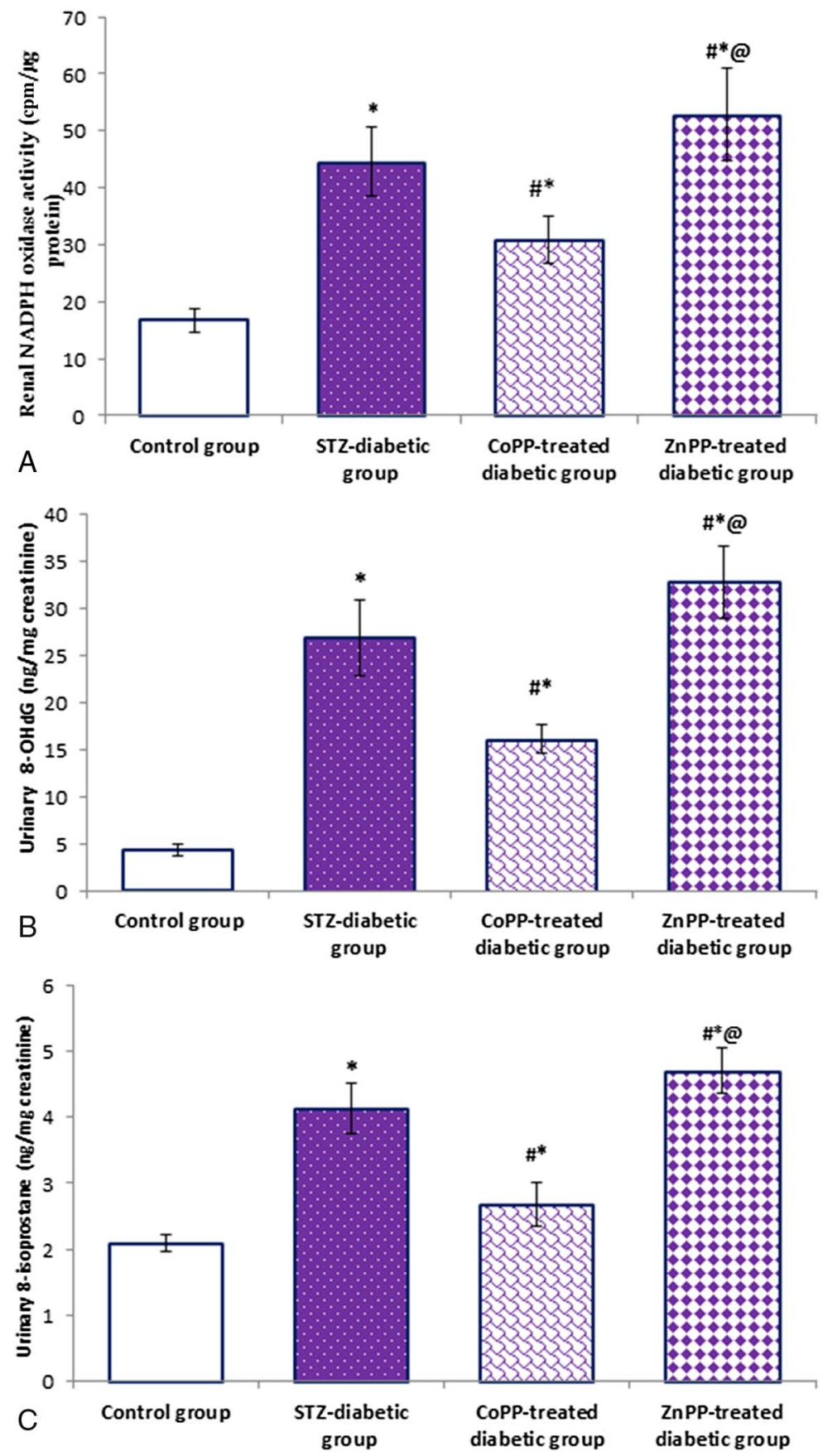

Fig. 4. Effects of the $\mathrm{HO}$ inducer, $\mathrm{CoPP}$ and $\mathrm{HO}$ inhibitor, $\mathrm{ZnPP}$ on renal oxidative stress markers, renal NADPH oxidase activity (A), urinary 8-isoprostane (B), and urinary 8-OHdG (C) in STZ-induced early DN in rats. Data are mean $\pm \mathrm{SD}$ of 10 rats. ${ }^{*} P<0.05$ vs. the control group; ${ }^{\#} P<0.05$ vs. the STZ-diabetic group; ${ }^{@} P<0.05$ vs. CoPPtreated diabetic group. $\mathrm{cpm} / \mu \mathrm{g}$ protein $=$ count per minute/microgram protein; 8 -OHdG $=8$-hydroxydeoxyguanosine 


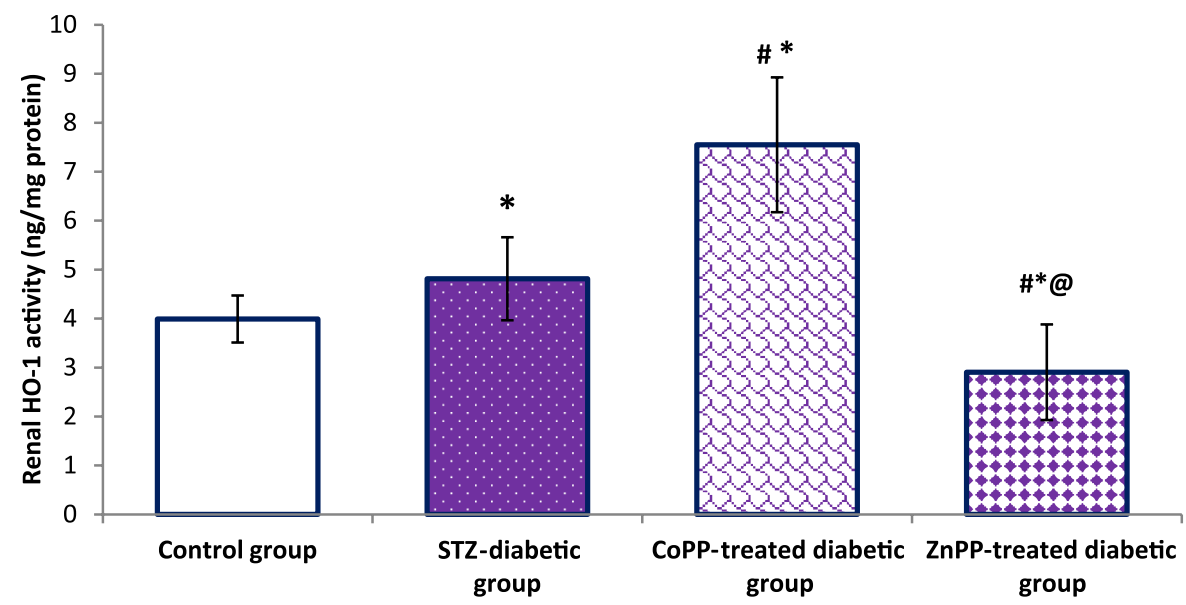

Fig. 5. Effects of the HO inducer, CoPP and HO inhibitor, ZnPP on renal HO-1 activity in STZ-induced early DN in rats. Data are mean \pm SD of 10 rats. ${ }^{*} P<0.05$ vs. the control group; ${ }^{\#} P<0.05$ vs. the STZ-diabetic group;

${ }^{\circledR} P<0.05$ vs. the CoPP-treated diabetic group

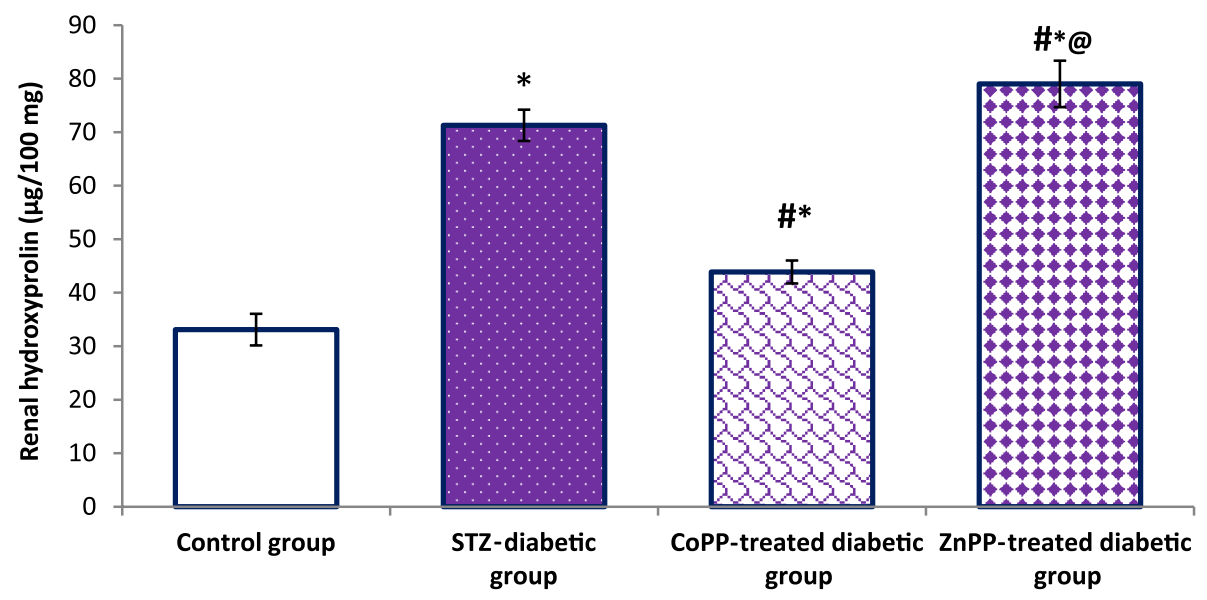

Fig. 6. Effects of the HO inducer, CoPP and HO inhibitor, ZnPP on renal hydroxyprolin level in STZ-induced early $\mathrm{DN}$ in rats. Data are mean $\pm \mathrm{SD}$ of 10 rats. ${ }^{*} P<0.05$ vs. the control group; ${ }^{\#} P<0.05$ vs. the STZ-diabetic group; ${ }^{\circledR} P<0.05$ vs. the CoPP-treated diabetic group

Zucker diabetic fatty rats (40), a genetically obese leptin receptor-deficient model (24), and STZ-induced diabetic rats (37).

Several possible mechanisms have been postulated to explain the antidiabetic effect of HO induction. Dong et al. (8) demonstrated that BR administration improved hyperglycemia and obesity by increasing insulin sensitivity. It has been widely reported that HO-1 upregulation protected pancreatic islet cells from apoptosis, and improved their functions in vivo (27). Moreover CoPP-induced HO-1 upregulation increases insulin release, possibly through CO (33), or through the abating c-jun N-terminal kinase (JNK) activity, which has been implicated in blocking insulin biosynthesis (19). 
The reduced hyperglycemia observed in CoPP-treated diabetic rats could be attributed to the CoPP-mediated increase of insulin secretion (Fig. 1), which would greatly improve glucose metabolism, through enhanced adenosine monophosphate-activated protein kinasedependent glucose transporter 4 (GLUT4) expression and translocation (15). The antidiabetic effect observed with CoPP could be due to potentiation of insulin-sensitizing pathways (22) through HO upregulation similarly to increases in the activity of other factors which also promote insulin signaling such as adiponectin $(22,37-40)$, cyclic guanosine monophosphate (cGMP), cyclic adenosine monophosphate (36), and peroxisome proliferatoractivated receptor $\alpha(36)$.

The concept that DN is essentially a micro-inflammatory disease and the role of microinflammation-mediated activation of intrinsic immune system has been recently emerging. The inflammatory cascade is triggered by the interactions between various chemokines secreted from resident glomerular cells such as MCP-1 and adhesion molecules such as ICAM-1, leading to the underlying pathological changes in DN (59). This observation has been confirmed using the ICAM-1 and MCP-1 gene knockout diabetic mice, as both exhibited markedly reduced kidney monocytes/macrophages accumulation, which was associated with lowered albuminuria and marked attenuation of renal injury (53).

Our findings indicated that MCP-1 and ICAM-1 levels were significantly increased in the kidneys of diabetic rats compared with the control group, while CoPP therapy markedly reduced their levels (Fig. 3), which may explain, at least in part, the beneficial effect of CoPP on DN.

In agreement with our results, Pan et al. (43), observed that ICAM-1 and MCP-1 expressions were increased in experimental diabetic renal injury, which were associated with inflammatory cell infiltration and adhesion as well as renal fibrosis.

Supporting the role of NF-kB activation and inflammation in DN, we found increased renal NF- $\kappa \mathrm{B}$ and TNF- $\alpha$ levels in diabetic rats. This is consistent with previous reports indicating that the expression of NF- B was significantly increased in the renal tissues of STZ-induced DN rats, and positively correlated with renal interstitial MCP-1 and ICAM-1 protein and mRNA expression and proteinuria (5).

The availability of binding sites for NF- $\kappa \beta$ on both the MCP-1 (65) and HO-1 gene promoters suggests greater interaction between this transcription factor and the HO system and inflammation (46). Supportive of this notion, our results of reduced NF- $\mathrm{kB}$ level in CoPP-treated animals was increased with blockade of the HO system with subsequently elevated inflammatory markers. This is consistent with previous reports indicating that HO-1 induction reduced the NF- $\kappa \mathrm{B}$-induced inflammation in GK and STZ-induced diabetic rats $(37,38)$.

The abrogated renal inflammatory markers observed in diabetic rats under CoPP therapy (Fig. 3) might be mediated by HO-induced inhibition of NF- $\mathrm{BB}$ activation. It has been previously demonstrated that NF- B blockage could be a major way of preventing diabetic renal damage by ablating the NF- B-induced inflammatory and oxidative stress in DN (30).

There were other supporting lines of evidence, in vivo (59) and in vitro (56), suggesting that the pathological actions of MCP-1 in DN could exceed its role in macrophage recruitment as MCP-1 could directly damage kidney cells or induce additional responses such as macrophage activation or the recruitment and activation of $\mathrm{T}$ cells.

TNF- $\alpha$ is cytotoxic to glomerular, mesangial and epithelial cells and competent to induce direct renal injury through the generation of reactive free radicals, through c-Src/ NADPH oxidase, which in turn initiates NF- $\kappa \mathrm{B}$ activation (57). 
Recently several potential mechanisms have been described to explain the metabolic effects of TNF- $\alpha$. TNF- $\alpha$ initiates the activation of caspase 8 via its binding to the death receptor TNF-R1 resulting in apoptosis (34).

Moreover TNF- $\alpha$ stimulates serine kinases, such as JNK and p38 mitogen-activated protein kinase (MAPK) resulting in serine phosphorylation of IRS-1 and IRS-2, which in turn reduces the downstream insulin signaling (41). Moreover, TNF- $\alpha$ induces insulin resistance by reducing the expression and translocation of GLUT4 by inhibiting the phosphatidylinositol 3 kinase/protein kinase B and peroxisome proliferator-activated receptor $\gamma$ signaling (36).

It is tempting to speculate that the HO-mediated suppression of TNF- $\alpha$ and NF- B constitutes not only an important anti-inflammatory mechanism to limit tissue damage in DN but also a mechanism that could be explored to improve insulin sensitivity and glucose metabolism.

The anti-inflammatory effect of HO-1 has also been highlighted in HO-1 deficient human cases and HO-1 knockout mice, where both exhibited a proinflammatory phenotype, with overexpression of several proinflammatory mediators, including MCP-1, ICAM-1, and NF-кB (20).

HO upregulation has been reported to modulate the inflammatory response by inhibiting the TNF- $\alpha$-induced monocyte adhesion to endothelia cells (54) or by inhibiting the expression of adhesion molecules, such as ICAM-1, in vitro and in vivo (60). The anti-inflammatory effect of $\mathrm{HO}$ upregulation could be mediated by depleting heme, which has several proinflammatory activities (14). The HO-1-based protection against allergic inflammation can involve $\mathrm{HO}-1$ by-products, such as $\mathrm{CO}$, which has been shown to mimic some protective actions of HO-1 (42). Rücker et al. (44) have shown that the expression of ICAM-1 was aggravated by selective inhibition of $\mathrm{HO}-1$ activity and was reversed by $\mathrm{HO}-1$ upregulation.

HO-1 upregulation is capable of modulating the polarization of macrophages toward the anti-inflammatory M2 phenotype, which effect results in a decrease of proinflammatory mediator release and an increase in the formation of anti-inflammatory mediators, such as IL-10. Consequently, the proinflammatory M1 phenotype and its related secretagogues like TNF- $\alpha$ and MCP-1 decrease, suggesting another anti-inflammatory mechanism of the HO-system (61).

We observed a significant increase in the levels of renal hydroxyproline content in STZinduced diabetic rats. Hydroxyproline increases the severity of the kidney damage and fibrosis in experimental animals, as it is demonstrated by deteriorated renal functions. Our data are consistent with those reported by Karihaloo (21).

Although the pathogenesis of tubular damage leading to tubulointerstitial fibrosis is undoubtedly multifactorial, it is generally accepted that oxidative stress is a principal mechanism. Activation of transcription factors and release of inflammatory markers leads to excessive collagen deposition in the diabetic kidney due to disruption of the balance between synthesis and degradation (4).

CoPP therapy effectively diminished the renal hydroxyproline level, suggesting its antifibrotic efficacy in diabetic conditions. The antifibrogenic properties of HO-1 and its products have been extensively studied. Increased HO activity in human hepatic myofibroblasts correlates with decreased proliferation and procollagen I mRNA expression, which was attributed to BR (26), while HO-1 inhibition in a rat hypoxia model increased collagen (type I, type III) and transforming growth factor beta-3 (TGF- $\beta 3$ ) expression, effects that could be attributed to decreased $\mathrm{CO}$ level (13). 
Our findings have indicated that CoPP-induced HO-1 upregulation not only plays a vital role in the modulation of the inflammatory process, but it also has an obvious antioxidant property through decreased renal NADPH oxidase activity, the mammalian enzyme dedicated to reactive oxygen species (ROS) generation (12), alongside with reduced urinary 8isoprostane and $8-\mathrm{OHdG}$ levels in diabetic rats.

Hyperglycemia is strongly associated with increased production of ROS, inducing DNA, protein, and lipid damage and triggering renal cell injury (17). Therefore, HO-1 upregulation, though having both hypoglycemic and antioxidant properties, might be considered a protective agent against DN.

The antioxidant effect of HO-1 has been confirmed by the phenotypic consequences of HO-1 deficient mice and a patient with HO-1 deficiency. HO-1 upregulation has been shown to decrease ROS and NADPH oxidase activity in vitro and in vivo. BR and Co have been suggested as potential mediators of the antioxidant effect of HO upregulation (32). Datla et al. (6) proved that upregulation of HO-1 gene expression was shown to decrease the availability of the heme-containing gp91 subunit necessary for NADPH oxidase activity.

The decreased podocyte number, mostly due to enhanced renal apoptosis, has been identified as a leading cause of proteinuria in DN (9).

In the current study, HO-1 decreased albuminuria in CoPP-treated diabetic rats. Several studies have suggested that one possible pathway by which HO-1 confers protection in $\mathrm{DN}$ is antiapoptotic activity that contributes to protecting diabetic rats from endothelial cell dysfunction by preventing endothelial cell sloughing (27), which is largely mediated by augmented iron efflux or CO (51). Indeed, HO induction has been proved to upregulate the expression of the antiapoptotic proteins p-AKT, BcL-XL, and p21 (16), and to decrease glomerular caspase 3 expression (7). At the same time, HO appears to reduce the proapoptotic effects of TNF- $\alpha$, hyperglycemia, and iron. At the intracellular level, this may involve expression of MAPK enzymes and possibly the activation of NF- B. (49).

Although several studies focused on HO-1 induction as a potential therapeutic target in DN, other works have directed attention to its product molecules as a protective strategy against injury. Increased HO-1 activity results in degradation of the heme moiety, a toxic pro-oxidant $(1,42)$. Indeed, BV and BR can directly scavenge ROS and inhibit lipid peroxidation $(2,28)$. In addition, ferritin is coinduced with HO-1, allowing sequestration of redox-active iron (11). Moreover, $\mathrm{CO}$ has vasodilator, anti-inflammatory and antiapoptotic effects (2) mediated via cGMP, and potassium channels (32). These signaling pathways exert broad-based, far ranging cellular effects that protect against renal damage in diabetes.

\section{Conclusion}

The studies on HO-1 in DN, including the one presented here, have demonstrated that HO-1 upregulation could exert effective protective role against STZ-induced early DN. Such protective effect was probably carried out by suppressing of the underlying inflammatory, pro-oxidant, and fibrotic pathways with improved glycemic control. The data support a therapeutic potential of HO-1 induction in DN that may open the way to translate such potential into a therapeutic reality. 


\section{REFERENCES}

1. Bak I, Czompa A, Juhasz B, Lekli I, Tosaki A: Reduction of reperfusion-induced ventricular fibrillation and infarct size via heme oxygenase-1 overexpression in isolated mouse hearts. J. Cell. Mol. Med. 14(9), 2268-2272 (2010)

2. Bak I, Szendrei L, Turoczi T, Papp G, Joo F, Das DK, de Leiris J, Der P, Juhasz B, Varga E, Bacskay I, Balla J, Kovacs P, Tosaki A: Heme oxygenase-1 related carbon monoxide production and ventricular fibrillation in isolated ischemic/reperfused mouse myocardium. FASEB J. 17, 2133-2135 (2003)

3. Bradford MM: Rapid and sensitive method for the quantitation of microgram quantities of protein utilizing the principle of protein-dye binding. Anal. Biochem. 72, 248-254 (1976)

4. Brosius FC: New insights into the mechanisms of fibrosis and sclerosis in diabetic nephropathy. Rev. Endocr. Metab. Disord. 9, 245-254 (2008)

5. Chen $\mathrm{P}$, Chen JB, Chen WY, Zheng QL, Wang YQ, Xu XJ: Effects of quercetin on nuclear factor- $\mathrm{kB}$ p65 expression in renal ubiquitin-proteasome system of diabetic rats. Zhonghua Nei Ke Za Zhi 51, 460-465 (2012)

6. Datla SR, Dusting GJ, Mori TA, Taylor C, Croft KD, Jiang F: Induction of heme oxygenase-1 in vivo suppresses NADPH oxidase derived oxidative stress. Hypertension 50, 636-642 (2007)

7. Di Noia MA, Van Driesche S, Palmieri F, Yang LM, Quan S, Goodman AI, Abraham NG: Heme oxygenase-1 enhances renal mitochondrial transport carriers and cytochrome $\mathrm{C}$ oxidase activity in experimental diabetes. J. Biol. Chem. 281(23), 15687-15693 (2006)

8. Dong H, Huang H, Yun X, Kim DS, Yue Y, Wu H, Sutter A, Chavin KD, Otterbein LE, Adams DB, Kim YB, Wang H: Bilirubin increases insulin sensitivity in leptin-receptor deficient and diet-induced obese mice through suppression of ER stress and chronic inflammation. Endocrinology 155(3), 818-828 (2014)

9. Elmarakby AA, Faulkner J, Baban B, Saleh MA, Sullivan JC: Induction of hemeoxygenase-1 reduces glomerular injury and apoptosis in diabetic spontaneously hypertensive rats. Am. J. Physiol. Renal Physiol. 302, F791-F800 (2012)

10. Elmarakby AA, Faulkner J, Pye C, Rouch K, Alhashim A, Maddipati KR, Baban B: Role of haem oxygenase in the renoprotective effects of soluble epoxide hydrolase inhibition in diabetic spontaneously hypertensive rats. Clin. Sci. (Lond.) 125, 349-359 (2013)

11. Farhangkhoee H, Khan ZA, Mukherjee S, Cukiernik M, Barbin YP, Karmazyn M, Chakrabarti S: Heme oxygenase in diabetes-induced oxidative stress in the heart. J. Mol. Cell. Cardiol. 35(12), 1439-1448 (2003)

12. Fu XJ, Peng YP, Hu YP, Shi YZ, Yao M, Zhang X: NADPH oxidase 1 and its derived reactive oxygen species mediated tissue injury and repair. Oxid. Med. Cell. Longev. 2014, 282854 (2014)

13. Gong LM, Du JB, Shi L, Shi Y, Tang CS: Effects of endogenous carbon monoxide on collagen synthesis in pulmonary artery in rats under hypoxia. Life Sci. 74, 1225-1241 (2004)

14. Graça-Souza AV, Arruda MA, de Freitas MS, Barja-Fidalgo C, Oliveira PL: Neutrophil activation by heme: implications for inflammatory processes. Blood 99, 4160-4165 (2002)

15. Holmes BF, Sparling DP, Olson AL, Winder WW, Dohm GL: Regulation of muscle GLUT4 enhancer factor and myocyte enhancer factor 2 by AMP-activated protein kinase. Am. J. Physiol. Endocrinol. Metab. 289(6), E1071-E1076 (2005)

16. Inguaggiato P, Gonzalez-Michaca L, Croatt AJ, Haggard JJ, Alam J, Nath KA.: Cellular overexpression of heme oxygenase-1 up-regulates p21 and confers resistance to apoptosis. Kidney Int. 60, 2181-2191 (2001)

17. Jiang F: NADPH oxidase in the kidney: a Janus in determining cell fate. Kidney Int. 75, 135-137 (2009)

18. Kanasaki K, Taduri G, Koya D: Diabetic nephropathy: the role of inflammation in fibroblast activation and kidney fibrosis. Front. Endocrinol. (Lausanne) 4, 7 (2013)

19. Kaneto H, Nakatani Y, Kawamori D, Miyatsuka T, Matsuoka TA, Matsuhisa M, Yamasaki Y: Role of oxidative stress, endoplasmic reticulum stress, and c-Jun N-terminal kinase in pancreatic $\beta$-cell dysfunction and insulin resistance. Int. J. Biochem. Cell Biol. 38, 782-793 (2006)

20. Kapturczak MH, Wasserfall C, Brusko T, Campbell-Thompson M, Ellis TM, Atkinson MA, Agarwal A: Heme oxygenase-1 modulates early inflammatory responses: evidence from the heme oxygenase-1-deficient mouse. Am. J. Pathol. 165, 1045-1053 (2004)

21. Karihaloo A: Anti-fibrosis therapy and diabetic nephropathy. Curr. Diab. Rep. 12(4), 414-422 (2012)

22. Kim DH, Burgess AP, Li M, Tsenovoy PL, Addabbo F, McClung JA, Puri N, Abraham NG: Heme oxygenasemediated increases in adiponectin decrease fat content and inflammatory cytokines tumor necrosis factor- $\alpha$ and interleukin-6 in Zucker rats and reduce adipogenesis in human mesenchymal stem cells. J. Pharmacol. Exp. Ther. 325, 833-840 (2008) 
23. Kim M, Moon H, Hong S: Determination of urinary 8-hydroxy-2- deoxy guanosine as a DNA damage marker. Am. Clin. Lab. 42-45 (2001)

24. Kuhlmann J, Neumann-Haefelin C, Belz U, Kalisch J, Juretschke HP, Stein M, Kleinschmidt E, Kramer W, Herling AW: Intramyocellular lipid and insulin resistance: a longitudinal in vivo $1 \mathrm{H}$-spectroscopic study in Zucker diabetic fatty rats. Diabetes 52, 138-144 (2003)

25. Lee JI, Ganster RW, Geller DA, Burckart GJ, Thomson AW, Lu L: Cyclosporine inhibits the expression of co-stimulatory molecules on in vitro generated dendritic cells: association with reduced nuclear translocation of nuclear factor kappa B. Transplantation 68, 1255-1263 (1999)

26. Li L, Grenard P, Nhieu JT, Julien B, Mallat A, Habib A, Lotersztajn S: Heme oxygenase-1 is an antifibrogenic protein in human hepatic myofibroblasts. Gastroenterology 125, 460-469 (2003)

27. Liu X, Wei J, Peng DH, Layne MD, Yet SF: Absence of heme oxygenase-1 exacerbates myocardial ischemia/ reperfusion injury in diabetic mice. Diabetes 54(3), 778-784 (2005)

28. Mancuso C, Santangelo R, Calabrese V: The heme oxygenase/biliverdin reductase system: a potential drug target in Alzheimer s disease. J. Biol. Regul. Homeost. Agents 27(2 Suppl.), 75-87 (2013)

29. Mann JFE, Rossing P, Więcek A, Rosivall L, Mark P, Mayer G: Diagnosis and treatment of early renal disease in patients with type 2 diabetes mellitus: what are the clinical needs? Nephrol. Dial. Transplant. 30(Suppl. 4), iv1-iv5 (2015)

30. Mezzano S, Aros C, Droguett A, Burgos ME, Ardiles L, Flores C, Schneider H, Ruiz-Ortega M, Egido J: NF-kappaB activation and overexpression of regulated genes in human diabetic nephropathy. Nephrol Dial. Transplant. 19, 2505-2512 (2004)

31. Milne GI, Sanchez SC, Musiek ES, Morrow JD: Quantification of F2-isoprostanes as a biomarker of oxidative stress. Nat. Protoc. 2, 211-226 (2007)

32. Mishra M, Ndisang JF: A critical and comprehensive insight on heme oxygenase and related products including carbon monoxide, bilirubin, biliverdin and ferritin in type-1 and type-2 diabetes. Curr. Pharm. Des. 20, 1370-1391 (2014)

33. Mosén H, Salehi A, Alm P, Henningsson R, Jimenez-Feltström J, Ostenson CG, Efendic S, Lundquist I: Defective glucose-stimulated insulin release in the diabetic Goto-Kakizaki (GK) rat coincides with reduced activity of the islet carbon monoxide signaling pathway. Endocrinology 146, 1553-1558 (2005)

34. Moujalled DM, Cook WD, Okamoto T, Murphy J, Lawlor KE, Vince JE, Vaux DL: TNF can activate RIPK3 and cause programmed necrosis in the absence of RIPK1. Cell Death Dis. 4, e465 (2013)

35. Mozaffari MS, Baban B, Liu JY, Abebe W, Sullivan JC, El-Marakby A: Mitochondrial complex I and NAD(P)H oxidase are major sources of exacerbated oxidative stress in pressure-overloaded ischemic-reperfused hearts. Basic Res. Cardiol. 106(2), 287-297 (2011)

36. Ndisang JF: Role of heme oxygenase in inflammation, insulin-signalling, diabetes and obesity. Mediators Inflamm. 2010, 359732 (2010)

37. Ndisang JF, Jadhav A: Heme oxygenase system enhances insulin sensitivity and glucose metabolism in streptozotocin-induced diabetes. Am. J. Physiol. Endocrinol. Metab. 296(4), E829-E841 (2009)

38. Ndisang JF, Jadhav A: Up-regulating the hemeoxygenase system enhances insulin sensitivity and improves glucose metabolism in insulin-resistant diabetes in Goto-Kakizaki rats. Endocrinology 150(6), 2627-2636 (2009)

39. Ndisang JF, Jadhav A, Mishra M: The heme oxygenase system suppresses perirenal visceral adiposity, abates renal inflammation and ameliorates diabetic nephropathy in Zucker diabetic fatty rats. PLoS One 9(1), e87936 (2014)

40. Ndisang JF, Lane N, Jadhav A: The heme oxygenase system abates hyperglycemia in Zucker diabetic fatty rats by potentiating insulin-sensitizing pathways. Endocrinology 150(5), 2098-2108 (2009)

41. Olson NC, Callas PW, Hanley AJ, Festa A, Haffner SM, Wagenknecht LE, Tracy RP: The insulin resistance atherosclerosis study. J. Clin. Endocrinol. Metab. 97(3), 1032-1040 (2012)

42. Pae HO, Kim EC, Chung HT: Heme oxygenase-1 and carbon monoxide: emerging therapeutic targets in inflammation and allergy. Recent Pat Inflamm. Allergy Drug Discov. 2, 159-165 (2008)

43. Pan Y, Zhang X, Wang Y, Cai L, Ren L, Tang L, Wang J, Zhao Y, Wang Y, Liu Q, Li X, Liang G: Targeting JNK by a new curcumin analog to inhibit NF-kB-mediated expression of cell adhesion molecules attenuates renal macrophage infiltration and injury in diabetic mice. PLoS One 8(11), e79084 (2013)

44. Rücker M, Schäfer T, Roesken F, Spitzer WJ, Bauer M, Menger MD: Reduction of inflammatory response in composite flap transfer by local stress conditioning-induced heat-shock protein 32. Surgery 129, 292-301 (2001)

45. Saleh MA, Boesen EI, Pollock JS, Savin VJ, Pollock DM: Endothelin-1 increases glomerular permeability and inflammation independent of blood pressure in the rat. Hypertension 56(5), 942-949 (2010) 
46. Sasaki T, Takahashi T, Maeshima K, Shimizu H, Toda Y, Morimatsu H, Takeuchi M, Yokoyama M, Akagi R, Morita K: Heme arginate pretreatment attenuates pulmonary NF- $\mathrm{KB}$ and AP-1 activation induced by hemorrhagic shock via heme oxygenase-1 induction. Med. Chem. 2, 271-274 (2006)

47. Sauvanet JP: Chronic kidney disease, an often underestimated complication of diabetes. Soins 794, S4-S11 (2015)

48. Sharma S, Kulkarni SK, Chopra K: Curcumin, the active principle of turmeric (Curcuma longa), ameliorates diabetic nephropathy in rats. Clin. Exp. Pharmacol. Physiol. 33, 940-945 (2006)

49. Silva G, Cunha A, Grégoire IP, Seldon MP, Soares MP: The antiapoptotic effect of heme oxygenase-1 in endothelial cells involves the degradation of p38 alpha MAPK isoform. J. Immunol. 177, 1894-1903 (2006)

50. Somania R, Singhai AK, Shivgunde P, Jain D: Asparagus racemosus Willd (Liliaceae) ameliorates early diabetic nephropathy in STZ induced diabetic rats. Indian J. Exp. Biol. 50, 469-475 (2012)

51. Song R, Kubo M, Morse D, Zhou Z, Zhang X, Dauber JH, Fabisiak J, Alber SM, Watkins SC, Zuckerbraun BS, Otterbein LE, Ning W, Oury TD, Lee PJ, McCurry KR, Choi AM: Carbon monoxide induces cytoprotection in rat orthotopic lung transplantation via anti-inflammatory and antiapoptotic effects. Am. J. Pathol. 163, 231-242 (2003)

52. Su N, Li HY, Huang MF, Jiang ZP, Zhou TB: Association of monocyte chemoattractant protein-1 2518G/A gene polymorphism with diabetic nephropathy risk. J. Recept. Signal Transduct. Res. 35, 94-97 (2015)

53. Su X, Chen X, Liu L, Chang X, Yu X, Sun K: Intracellular adhesion molecule-1 K469E gene polymorphism and risk of diabetic microvascular complications: a meta-analysis. PLoS One 8(7), e69940 (2013)

54. Sun X, Pi J, Liu W, Hudson LG, Liu KJ, Feng C: Induction of heme oxygenase 1 by arsenite inhibits cytokine induced monocyte adhesion to human endothelial cells. Toxicol. Appl. Pharmacol. 236, 202-209 (2009)

55. Tang SC, Leung J, Lai KN: Diabetic tubulopathy: an emerging entity. Contrib. Nephrol. 170, 124-134 (2011)

56. Tesch GH, Schwarting A, Kinoshita K, Lan HY, Rollins BJ, Kelley VR: Monocyte chemoattractant protein-1 promotes macrophage-mediated tubular, but not glomerular injury, in nephrotoxic serum nephritis. J. Clin. Invest. 103, 73-80 (1999)

57. Therrien FJ, Agharazii M, Lebel M, Larivière R: Neutralization of tumor necrosis factor-alpha reduces renal fibrosis and hypertension in rats with renal failure. Am. J. Nephrol. 36(2), 151-161 (2012)

58. Varley H (1980): Non-protein nitrogen, urea, urate, creatine and creatinine. In: Practical and Clinical Biochemistry, ed Varley H, White Friars Press, London, pp. 452-488

59. Virella G, Lopes-Virella MF: The role of the immune system in the pathogenesis of diabetic complications. Front. Endocrinol. (Lausanne) 5, 126 (2014)

60. Wagener F, da Silva JL, Farley T, de Witte T, Kappas A, Abraham NG: Differential effects of heme oxygenase isoforms on heme mediation endothelial intracellular adhesion molecule 1 expression. J. Pharmacol. Exp. Ther. 291, 416-423 (1999)

61. Weis N, Weigert A, von Knethen A, Brüne B: Heme oxygenase-1 contributes to an alternative macrophage activation profile induced by apoptotic cell supernatants. Mol. Biol. Cell 20, 1280-1288 (2009)

62. Woessner JF: The determination of hydroxyproline in tissue and protein samples containing small proportions of this imino acid. Arch. Biochem. Biophys. 93, 440-447 (1961)

63. Wu H, Kong L, Zhou S, Cui W, Xu F, Luo M, Li X, Tan Y, Miao L: The role of microRNAs in diabetic nephropathy. J. Diabetes Res. 2014, 920134 (2014)

64. Yi B, Hu X, Zhang H, Huang J, Liu J, Hu J, Li W, Huang L: Nuclear NF-kB p65 in peripheral blood mononuclear cells correlates with urinary MCP-1, RANTES and the severity of type 2 diabetic nephropathy. PLoS One 9(6), e99633 (2014)

65. Zhang Z, Yuan W, Sun L, Szeto FL, Wong KE, Li X, Kong J, Li YC: 1, 25-Dihydroxy vitamin D3 targeting of NF- $\kappa$ B suppresses high glucose-induced MCP-1 expression in mesangial cells. Kidney Int. 72, 193-201 (2007)

66. Zhou D, Brown SA, Yu T, Chen G, Barve S, Kang BC, Thompson JS: A high dose of ionizing radiation induces tissue specific activation of nuclear factor- $\mathrm{B}$ in vivo. Radiat. Res. 151, 703-709 (1999) 